\title{
Preparation and characterization of multifunctional free-standing Ni/epoxy composite films
}

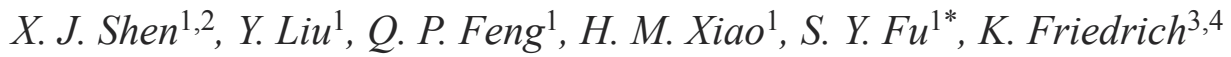 \\ ${ }^{1}$ Technical Institute of Physics and Chemistry, Chinese Academy of Sciences, 100190 Beijing, China \\ ${ }^{2}$ Graduate School, Chinese Academy of Sciences, 100039 Beijing, China \\ ${ }^{3}$ Institute for Composite Materials (IVW GmbH), Technical University of Kaiserslautern, 67663 Kaiserslautern, Germany \\ ${ }^{4}$ CEREM, King Saud University, Riyadh, Saudi Arabia
}

Received 12 April 2012; accepted in revised form 8 June 2012

\begin{abstract}
A new method is reported on preparation of multifunctional free-standing Ni/epoxy composite films with comprehensive physical properties under an applied magnetic field. A water soluble poly(vinyl alcohol) film is used as an interlayer film to separate epoxy resin and glass wafer. Ultrafine Ni particles are incorporated into a self-designed flexible-type transparent epoxy resin to get composite films. For the purpose of comparison, random Ni/epoxy composite films are also prepared in the absence of the applied magnetic field. The aligned composite films are endowed with promising optical, mechanical, electrical, and ferromagnetic properties. The specific resistance is $4-9$ orders lower in the vertical direction than that in the horizontal plane and the squareness ratio in the vertical direction is about $50 \%$ higher than in the horizontal plane. Meanwhile, their transmittance is much higher than that of the random composites. The anisotropies in their electrical and ferromagnetic properties are very useful material characteristics that may be explored for many applications.
\end{abstract}

Keywords: polymer composites, electrical conductivity, ferromagnetic property, transmittance

\section{Introduction}

With the rapid development of modern sciences and technologies, new materials with comprehensive physical properties are desired to meet the requirements of multifunctional devices [1-4]. Especially, optically transparent, mechanically flexible but electrically conductive and ferromagnetic material films are very useful in practical applications for flexible multifunctional devices in various electronic and electro-optical $[5,6]$, magneto-optical recording $[7,8]$, surface mount $[9,10]$ and new sensor technologies $[11,12]$. Polymer based composites are nowadays more and more considered as promising candidates for this kind of applications [13-15].

Transparent polymers such as poly(vinyl alcohol) (PVA) and epoxy resin, etc. can be made to be mechanically flexible $[16,17]$, but they are completely electrical insulators and nonmagnetic materials. In order to make them electrically conductive and ferromagnetic, functional particles such as $\mathrm{Ni}$ and $\mathrm{Co}$, etc. must be incorporated to form microand nano-composites [13, 15, 18-20]. Thermosetting polymers have the advantages such as ease of processability and low cost of production, etc. Epoxy resins are a class of extensively used thermosetting polymers and their multifunctional composites have wide applications in surface mount, space and electronic technologies [14, 15, 21]. Unfortunately, the resultant composites usually become opaque by the introduction of functional particles. This is due to the fact that the refractive index mismatches between organic polymers and inorganic particles [22]. It is thus of great significance to

\footnotetext{
${ }^{*}$ Corresponding author, e-mail: syfu@mail.ipc.ac.cn
}

(c) BME-PT 
develop transparent conductive and ferromagnetic composites based on epoxy resins. However, little work has been reported yet on transparent epoxy composite films with comprehensive physical properties. Moreover, free standing films have some advantages such as easy operation and flexibility in practical applications for multifunctional devices. It is still a great challenge to develop free standing composite films based on epoxy resins since epoxy resins are strongly adhesive to various substrates [23-25].

Epoxy and its composite films can be prepared on various substrates [15, 26-28]. Epoxy films were synthesized by coating epoxy polymer on low carbon steel substrates degreased with organic solvents $[26,27]$. Epoxy-polyester films were prepared on stainless steel substrate by electrostatic spray deposition [28]. The magnetic conductor micro-sized $\mathrm{Ni}$ powders and filaments were added to the epoxy matrix and then mixed thoroughly, finally the composite films were then obtained on a flat glass substrate [15]. However, it is hard to prepare free-standing epoxy composite films as mentioned above. In order to prepare free-standing epoxy composite films, release oil agent or polytetrafluoroethylene (PTFE) can be used to assist in peeling off epoxy composite films from substrates, but it is hard to form complete and good quality epoxy composite films due to large contact angles of epoxy resins with release oil agent or PTFE. Thus, it is of great importance to prepare free-standing epoxy composite films via a new approach.

In this work, a new method is developed for successful preparation of free-standing epoxy composite films. One key point here is that a water soluble PVA film is used as the interlayer film to separate epoxy resin and glass substrate. The PVA film has not only a small contact angle with epoxy resin but can also be water-soluble so that complete and high quality epoxy and composite films can be easily formed. In order to create transparent composite films, the as-prepared ultrafine monodisperse $\mathrm{Ni}$ particles with uniform sizes are aligned in the epoxy matrix by an applied magnetic field to allow light to transmit in the vertical direction. To get flexible epoxy and composite films, brittle epoxy resins can be modified using flexible modifiers [29-32]. This was realized by adding a flexible diamine to the epoxy resin matrix [33]. The aligned composites showed obvious anisotropies in electrical and ferromagnetic properties and such anisotropies are very useful material characteristics that can be exploited for many applications [13-15, 34, 35]. For the purpose of comparison and as reference materials, the random composite films were also prepared by a conventional direct mixing method.

\section{Experimental}

\subsection{Materials}

Ultrafine Ni particles with a diameter of about $114 \mathrm{~nm}$ were synthesized in our laboratory via a simple surfactant-free solvothermal method [36]. The matrix used was a transparent epoxy resin based on bisphenol-A (WS615) with an epoxy value of 0.500.56 , purchased from Wuxi Resin Factory, Wuxi, China. The hardener was composed of flexible diamine (Jeffamine D-230, molecular weight $M_{\mathrm{w}}=$ 230 , amine equivalent weight $=57.5$ ) obtained from Huntsman Chemical Co., Salt Lake City, Utah, USA and alicyclic amine WK-6822 (amine value $=$ $260 \pm 10$ ) obtained from Wells Sheng Intl. Trading Co., Guangzhou, China. $\gamma$-glycidoxypropyltrimethoxysilane (KH-560) bought from Nanjing \& Compton Co. Nanjing, China, was used as coupling agent. Flexible diamine (D-230) was used to modify the epoxy resin in order to get flexible epoxy composite films after curing [33]. The water soluble polyvinyl alcohol (PVA) film (Q-type) purchased from Yongan SYF Water Soluble Films Co., Ltd., Yongan, China was employed as the interlayer film to separate epoxy resin and glass substrate for an easy preparation of free-standing epoxy composite films.

\subsection{Preparation of free-standing composite films}

The compositions for preparing the composite films are shown in Table 1. Firstly, ultrafine monodisperse Ni particles, coupling agent and hardener were mixed in a conical flask and sonicated for $20 \mathrm{~min}$. Then, the equivalent amount of the epoxy resin was mixed with the above mixture by ultrasonic treatment for $10 \mathrm{~min}$. Afterwards, the suspension was degassed with a vacuum pump to eliminate air bubbles. When the air bubbles were sufficiently removed, $0.3 \mathrm{~g}$ of the resultant mixture was spread on a water soluble PVA film which was precoated on a glass slide substrate coated with vac- 
Table 1. Formulations for preparation of epoxy and Ni/epoxy composite films

\begin{tabular}{|l|c|c|c|c|c|c|c|}
\hline Sample code & $\begin{array}{c}\text { Ni weight } \\
{[\mathbf{g}]}\end{array}$ & $\begin{array}{c}\text { Ni content } \\
{[\mathbf{w t} \% \mathbf{l}]}\end{array}$ & $\begin{array}{c}\text { Bisphenol-A (WS615) } \\
\mathbf{[ g}]\end{array}$ & $\begin{array}{c}\mathbf{D}-230 \\
{[\mathbf{g}]}\end{array}$ & $\begin{array}{c}\text { WK6822 } \\
{[\mathbf{g}]}\end{array}$ & $\begin{array}{c}\text { KH-560 } \\
{[\mathbf{g}]}\end{array}$ & $\begin{array}{c}\text { Applied } \\
\text { magnetic field }\end{array}$ \\
\hline Pure epoxy & - & - & 3.10 & 0.60 & 0.60 & 0.11 & - \\
\hline $\mathrm{A}$ & 0.02 & 0.5 & 3.10 & 0.60 & 0.60 & 0.11 & no \\
\hline $\mathrm{B}$ & 0.09 & 2.0 & 3.10 & 0.60 & 0.60 & 0.11 & no \\
\hline $\mathrm{C}$ & 0.28 & 6.0 & 3.10 & 0.60 & 0.60 & 0.11 & no \\
\hline $\mathrm{A}_{\mathrm{m}}$ & 0.02 & 0.5 & 3.10 & 0.60 & 0.60 & 0.11 & yes \\
\hline $\mathrm{B}_{\mathrm{m}}$ & 0.09 & 2.0 & 3.10 & 0.60 & 0.60 & 0.11 & yes \\
\hline $\mathrm{C}_{\mathrm{m}}$ & 0.28 & 6,0 & 3.10 & 0.60 & 0.60 & 0.11 & yes \\
\hline
\end{tabular}
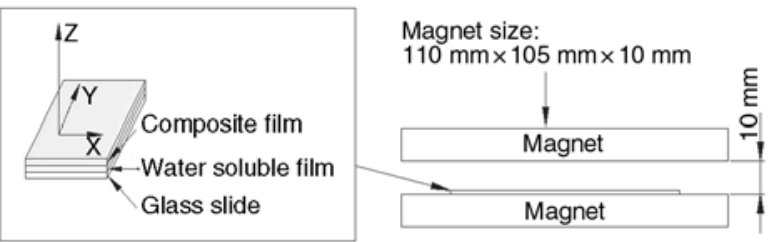

Figure 1. Schematic of the fixture for the curing of the composite samples under the applied magnetic field

uum silicon grease. The use of such a PVA interlayer film was necessary to easily obtain free-standing epoxy and composite films. Without using a PVA film as an interlayer, the epoxy resin would strongly adhere to the glass, making it very difficult to be free-standing.

The experimental fixture consisted of two permanent magnets in order to apply a magnetic field during the curing of the composites, as illustrated in Figure 1. The magnets with the pole section area of $110 \mathrm{~mm} \times 105 \mathrm{~mm}$ and the pole-to-pole gap of $10 \mathrm{~mm}$ could produce a magnetic field of ca. $63680 \mathrm{~A} / \mathrm{m}$. The samples were cured at room temperature for $6 \mathrm{~h}$, and then $80^{\circ} \mathrm{C}$ for $4 \mathrm{~h}$ in the presence of the applied magnetic field. When the samples were cooled down to room temperature, the composite films along with the water soluble film were peeled off from the glass substrates. They were then placed in an $80^{\circ} \mathrm{C}$ hot water till the water soluble film was completely dissolved. Finally, the high-quality free-standing composite films of about 150-200 $\mu$ m thickness were readily prepared. For the purpose of comparison, the samples were also prepared in the absence of the applied magnetic field under the same curing condition. Thus, the samples with three different $\mathrm{Ni}$ contents of $0.5,2.0$ and $6.0 \mathrm{wt} \%$ were synthesized.

\subsection{Characterization and measurement}

Scanning electron microscope (SEM) images were obtained to characterize the morphology and dispersion of the ultrafine Ni particles, using a Hitachi S-4300 microscope (Hitachi Co., Ltd., Tokyo,
Japan). The phase purity of the products was determined by X-ray diffraction (XRD) (D8 focus, Bruker Co., Karlsruhe, Germany) with a $\mathrm{Cu} \mathrm{K} \alpha$ radiation $(\lambda=1.5418 \AA)$. Top- and side-view micrographs were taken by an optical polarized microscope (Leica DM 2500M with an attached digital camera, DH-HV3100FC, Leica Microsystems Co., Wetzlar, Germany). The side-view samples were prepared by cryo-fracture via liquid nitrogen. The transmittance spectra of the films were detected using a UV-Vis spectrophotometer (U-3900, Hitachi Co., Ltd., Tokyo, Japan). The DC specific resistance was measured by a high-resistance \& microcurrent meter (EST121, Beijing Institute of Labor Protection, Beijing, China). For the electrical conductivity measurement, the sample surfaces were pasted by silver paste with an area of $5 \mathrm{~mm} \times 5 \mathrm{~mm}$ to ensure a perfect electrical contact. The area of the silver paste was large enough and thus the measured conductivity was independent of the contact area of the silver paste. Three measurements of the conductivity were conducted for each sample. The magnetic measurement was carried out at room temperature for the as-prepared samples, using a vibrating sample magnetometer (VSM 7307, Lakeshore Co., Westerville, USA) with a maximum magnetic field of $796 \mathrm{kA} / \mathrm{m}$. The contact angle was measured by an automatic contact angle meter (SL200B, Solon Information \& Technology Co., Shanghai, China).

\section{Results and discussion}

\subsection{Characterization of Ni particles and contact angle of epoxy with base substrate materials}

Ultrafine monodisperse Ni particles with a diameter of $113.7 \pm 7.1 \mathrm{~nm}$ were synthesized via a solvothermal method at a reaction temperature of $100^{\circ} \mathrm{C}$ for $3 \mathrm{~h}$ [36]. Figure 2a illustrates the as-synthesized spherical Ni particles. The size of the Ni particles 


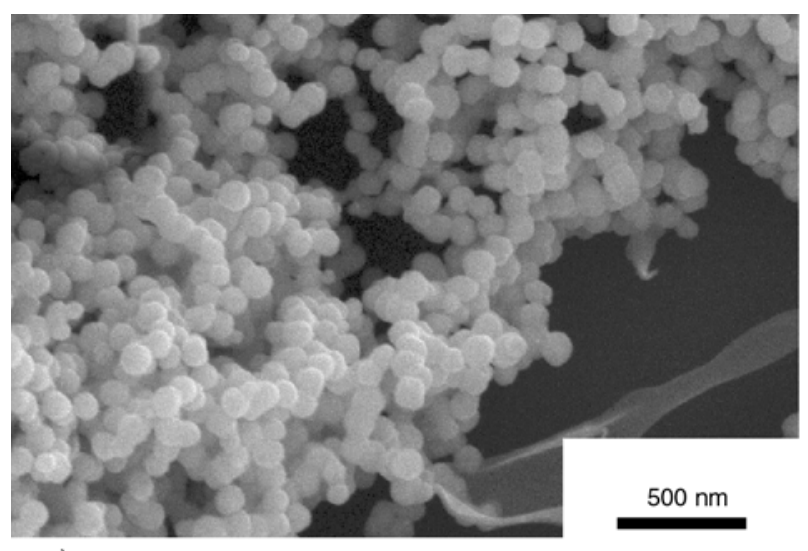

a)

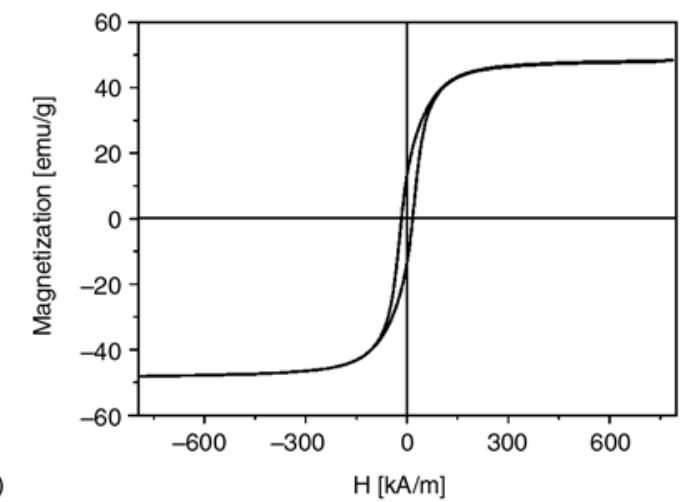

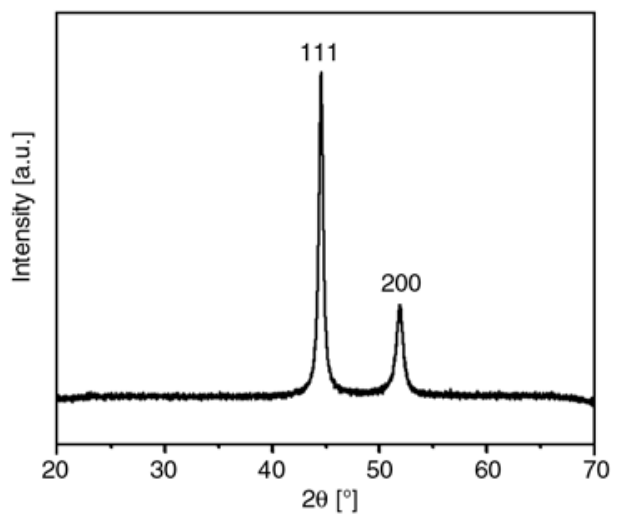

b)

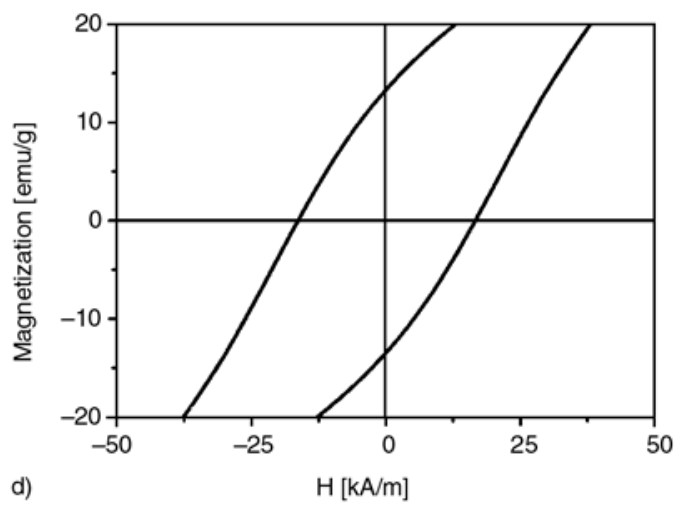

Figure 2. (a) SEM image, (b) XRD pattern and (c) hysteresis loops at room temperature of the as-prepared ultrafine nickel particles as well as (d) enlarged part of (c)

prepared under such a reaction condition showed no dependence on the reaction time [36], i.e. the following polymerization will not affect the size of the $\mathrm{Ni}$ particles. The X-ray powder diffraction (XRD) pattern of the ultrafine Ni particles is displayed in Figure $2 b$. The two peaks detected for the Ni sample are assigned to diffractions from the (111) and (200) planes of the face-centered cubic (fcc) $\mathrm{Ni}$ structure, respectively [36]. This is consistent with the values in the standard card (JCPDS card No. 4485). No impurity peaks were detected in the experimental range, thus indicating the formation of pure Ni particles. The magnetic hysteresis measurement of the ultrafine Ni particles was carried out at room temperature in an applied magnetic field, sweeping from -796 to $796 \mathrm{kA} / \mathrm{m}$. Figure $2 \mathrm{c}$ and $2 \mathrm{~d}$ shows the room temperature magnetic hysteresis curves of the as-prepared samples. The ultrafine Ni particles show ferromagnetic behavior with a saturation magnetization $\left(M_{\mathrm{s}}\right)$ of about $48.24 \mathrm{emu} / \mathrm{g}$ and a remanence $\left(M_{\mathrm{r}}\right)$ of about $13.39 \mathrm{emu} / \mathrm{g}$. Meanwhile, they have a relatively high coercivity $(\mathrm{Hc})$ of $16484 \mathrm{~A} / \mathrm{m}$, compared with the coercive force value $(56 \mathrm{~A} / \mathrm{m})$ of the bulk one at room temperature [37].
In order to show the advantages of the PVA interlayer film over normally used PTFE or release oil agents for preparing epoxy films, the contact angles of the epoxy resin with these base materials were measured. The contact angles of epoxy resin with PTFE or release oil coated glass substrate were obtained to be 45.4 and 38.6 degrees, respectively. In contrast, the contact angle with the water soluble film was much smaller, amounting to just an angle of 17.6 degrees. Hence, it is much easier to form epoxy and composite films based on the water soluble PVA film than based on the PTFE or release oil agents. If the epoxy was spread directly on a glass substrate, the formed epoxy film could not be peeled off from the substrate due to the strong adhesion between the epoxy and the glass. Therefore, the advantage of the present work is that there is no need of peeling off the epoxy composite films from the water soluble PVA interlayer film, but just by simply dissolving the water soluble PVA substrate.

\subsection{Transmittance of Ni/epoxy composite films}

The top-view and side-view optical micrographs of both the aligned and random $2.0 \mathrm{wt} \% \mathrm{Ni} /$ epoxy 


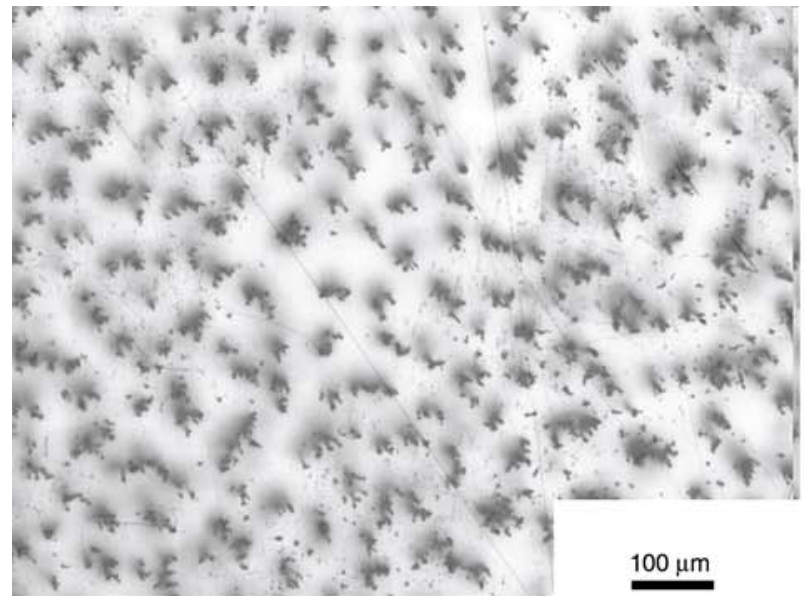

a)

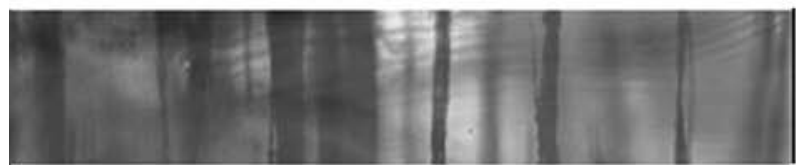

$100 \mu \mathrm{m}$

c)

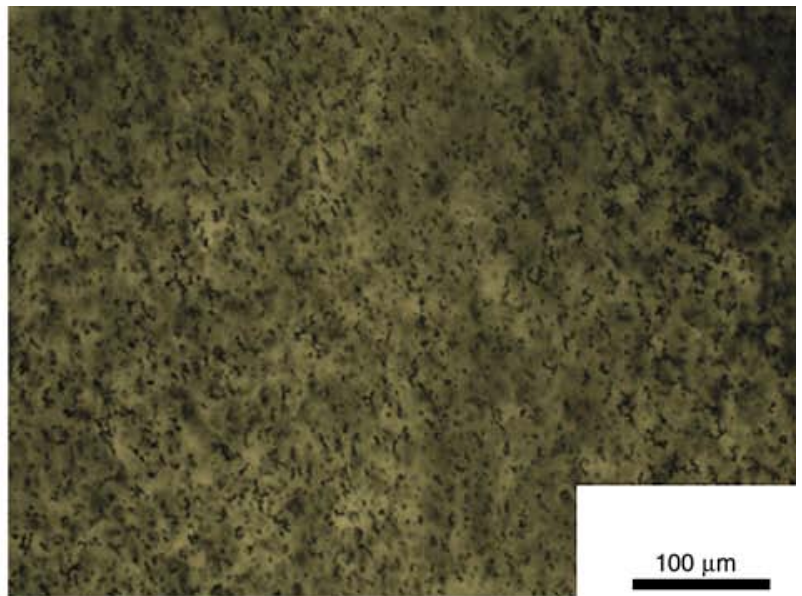

b)

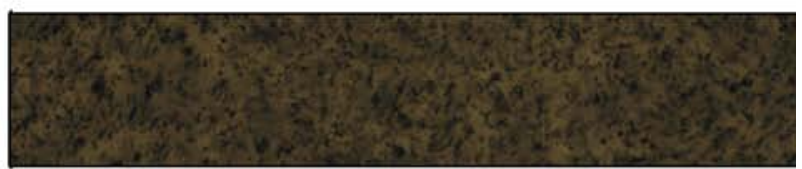

$100 \mu \mathrm{m}$

d)

Figure 3. (a, b) Top-view (along the $z$-axis shown in Figure 1) and (c, d) side-view optical micrographs of the Ni/epoxy composite films (left $\mathrm{B}_{\mathrm{m}}$ and right $\mathrm{B}$ )

composites are displayed in Figure 3. It reveals that the Ni columns in the composite medium are aligned along the magnetic field, and they are separated and spaced from each other (Figure 3a and 3c).

When the ultrafine Ni particles are uniformly dispersed in the epoxy matrix and subjected to a unidirectional magnetic field, they are aligned into a chain configuration to minimize the magnetostatic energy. Because they are in a fluid epoxy medium before curing, the Ni particles will move to minimize the potential energy of the system. The resultant magnetic dipoles in the neighboring columns repel each other, thus positioning the columns towards an equilibrium spacing [14]. The surface tension of the epoxy matrix prevents the undesirable overgrowth of the Ni columns beyond the thickness of the layer. The magnetically aligned conductive structure is retained after the epoxy matrix is cured. Moreover, most of the columns extend throughout the whole thickness of the composite film. Figure $3 b$ and $3 d$ show the top-view and side-view optical micrographs of the composite medium prepared in the absence of the applied magnetic field. As predicted, the ultrafine Ni particles were distributed randomly

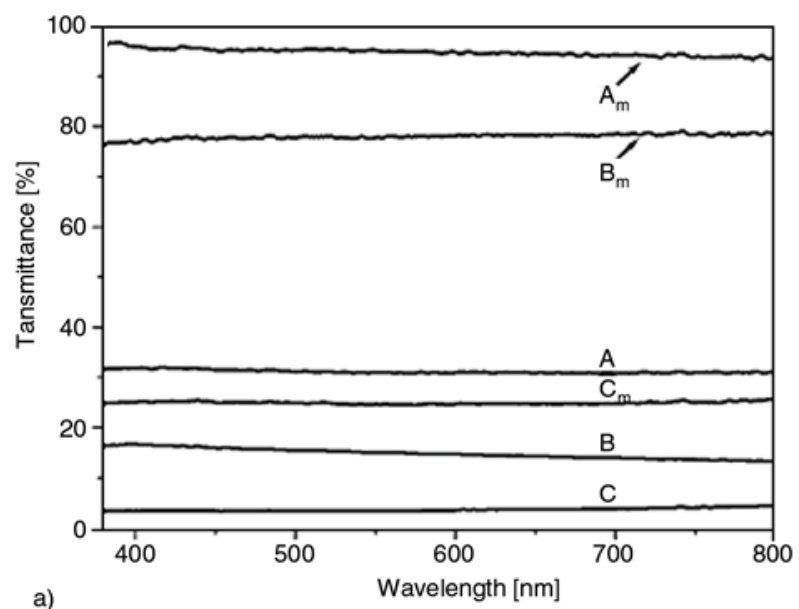

\begin{tabular}{|ccc|c|c|c|c}
\hline TIPC & TIPC & TIPC & TIPC & TIPC & $\mathrm{C}_{m}$ & TIPC \\
TIPC & TIPC & TIPC & TIPC & TIPC & TIPC & TIPC \\
TIPC & TIPC & TIPC & TIPC & TIPC & IIPC & TIPC \\
\hline TIPC & TIPC & TIPC & \multirow{2}{*}{ TIPC } & TIPC \\
& A & & B & & T & TIPC \\
TIPC & TIPC & TIPC & & TIPC & & TIPC \\
TIPC & TIPC & TIPC & & TIPC & & TIPC \\
\hline
\end{tabular}

b)

Figure 4. (a) UV-vis transmittance spectra of the Ni/epoxy composite films (calibrated against the pure epoxy film) along the vertical direction, and (b) digital photographs of the Ni/epoxy composite samples 
in the epoxy matrix. This would bring about an opaque composite medium, which is confirmed in Figure 4.

Figure $4 \mathrm{a}$ shows the effect of the Ni alignment on the transmittance and transparency of the composite films with different Ni contents, whereby the data were calibrated against a pure epoxy sample. It can be seen that the transmittance of the composite films is dramatically improved by the alignment of the ultrafine Ni particles induced under the applied magnetic field. Therefore, a relatively higher transmittance and transparency have been observed for the aligned composites than for the random composites with the same Ni contents. However, there will be increased light scattering and blockage for both the random and aligned composites as the $\mathrm{Ni}$ particle content increases. Besides, an altered refractive index in the strained polymer material near the ultrafine Ni particles will also cause a loss in transmittance [14]. Therefore, a higher Ni content will lead to more severe light scattering and blockage, and the transmittance of the composite films decreases dramatically as the Ni content increases (Figure 4a). The corresponding transparency shown in Figure $4 \mathrm{~b}$ confirms this tendency.

\subsection{Electrical resistance and mechanical flexibility of $\mathrm{Ni}$ /epoxy composite films}

The epoxy composites consisted of epoxy and ferromagnetic ultrafine $\mathrm{Ni}$ particles. The ultrafine $\mathrm{Ni}$ particles created aligned columns along the applied magnetic field, as shown in Figure 3a and 3c. The ferromagnetic ultrafine Ni particles are magnetized under the applied magnetic field, and these magnetized particles interact with adjacent particles due to their polarity. Finally, a columned structure of ultrafine $\mathrm{Ni}$ particles is formed due to repulsion of $\mathrm{Ni}$ particles in the as-prepared composites after they are cured. As a result, the current will primarily

Table 2. Specific resistance of the pure epoxy film and the $\mathrm{Ni}$ /epoxy composite films

\begin{tabular}{|l|c|c|}
\hline Sample code & $\begin{array}{c}\text { Specific resistance of } \\
\text { the horizontal plane } \\
{[\mathbf{\Omega} \cdot \mathbf{c m}]}\end{array}$ & $\begin{array}{c}\text { Specific resistance of } \\
\text { the vertical direction } \\
{[\mathbf{\Omega} \cdot \mathbf{c m}]}\end{array}$ \\
\hline Pure epoxy film & $8.4 \cdot 10^{14}$ & $9.5 \cdot 10^{14}$ \\
\hline $\mathrm{A}$ & $4.8 \cdot 10^{14}$ & $6.0 \cdot 10^{14}$ \\
\hline $\mathrm{B}$ & $2.3 \cdot 10^{13}$ & $1.6 \cdot 10^{13}$ \\
\hline $\mathrm{C}$ & $4.6 \cdot 10^{12}$ & $3.4 \cdot 10^{12}$ \\
\hline $\mathrm{A}_{\mathrm{m}}$ & $3.8 \cdot 10^{12}$ & $6.8 \cdot 10^{10}$ \\
\hline $\mathrm{B}_{\mathrm{m}}$ & $5.5 \cdot 10^{13}$ & $2.4 \cdot 10^{5}$ \\
\hline $\mathrm{C}_{\mathrm{m}}$ & $5.9 \cdot 10^{13}$ & $2.3 \cdot 10^{4}$ \\
\hline
\end{tabular}

flow along the direction of the applied magnetic field. To demonstrate this fact, the electrical conductivity in both the vertical ( $z$-axis) direction and horizontal plane was measured. For the vertical measurement, the composite film was sandwiched between two copper electrodes, with a thin layer of silver paste at the interface between the copper electrodes

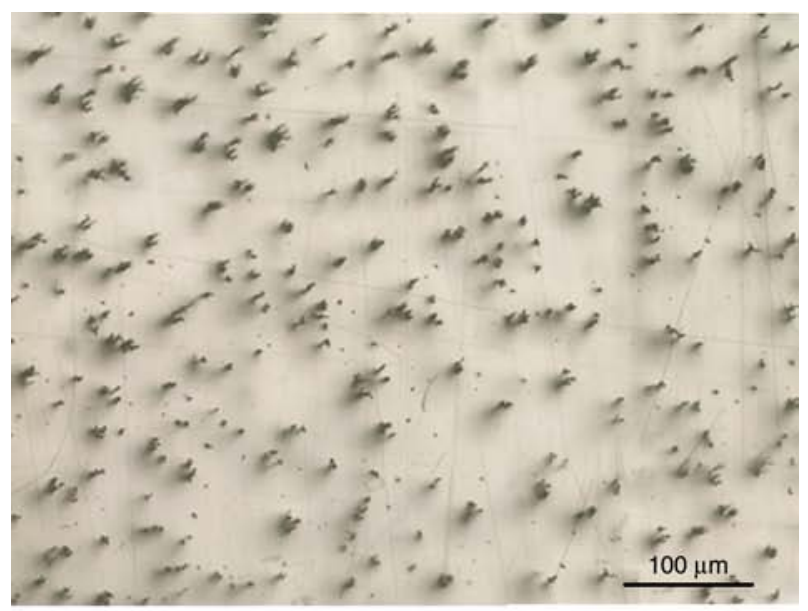

a)

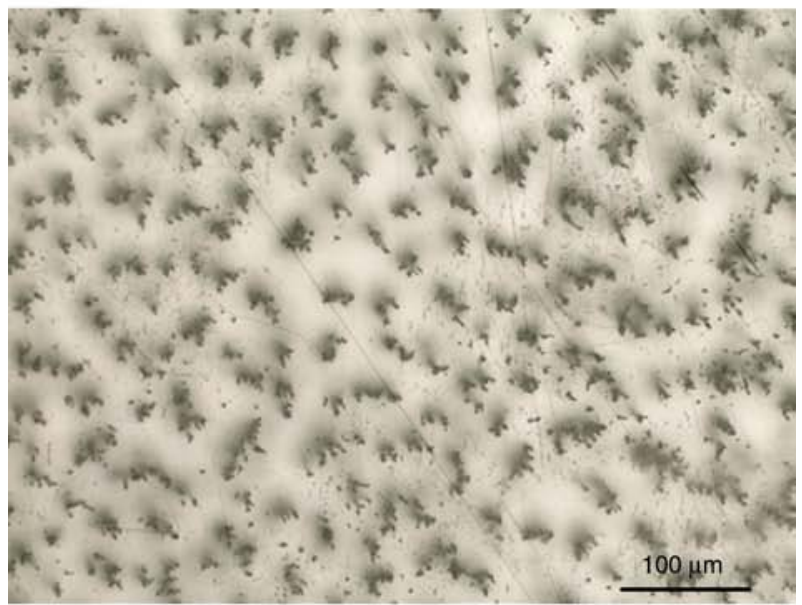

b)

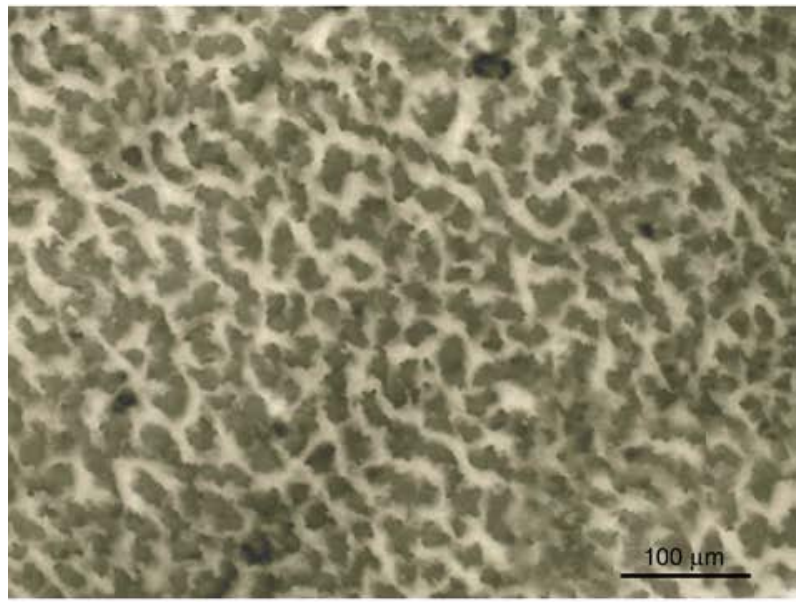

c)

Figure 5. Top-view optical micrographs of the aligned Ni/epoxy composites: (a) $A_{m}$, (b) $B_{m}$ and (c) $C_{m}$ 


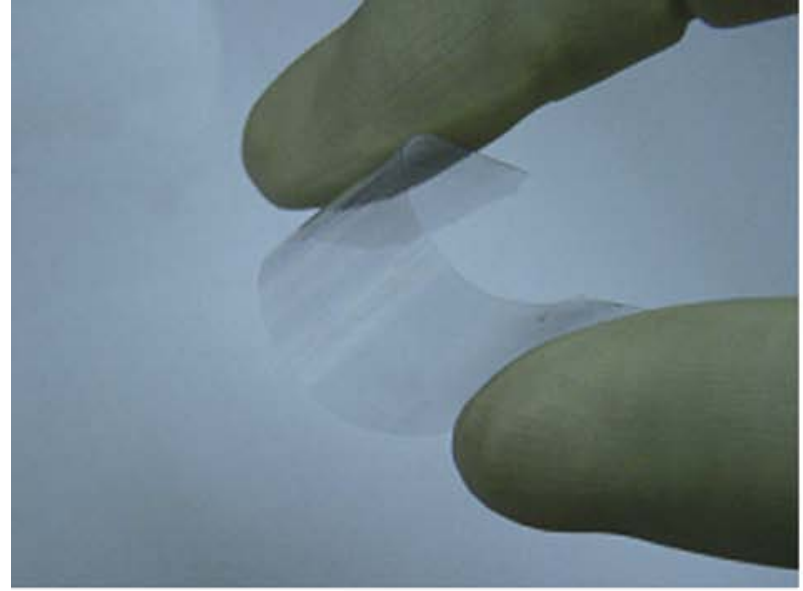

a)

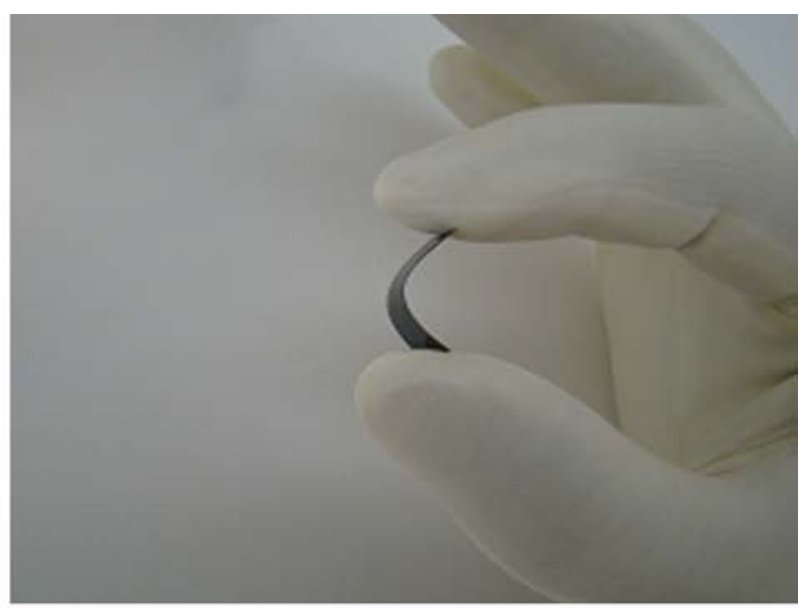

b)

Figure 6. Digital photographs of the composite films bent by fingers: (a) $A_{m}$ and (b) $C_{m}$

and the composite film to reduce the contact resistance. The horizontal electrical property of the composites was measured directly by two copper electrodes which clamp the two ends of the sample, respectively. All the electrical properties of the composites were measured at room temperature.

The corresponding results for the specific resistance are presented in Table 2. It can be seen that the specific resistance of the composite films decreases as the Ni content increases. This is easy to understand since the distance between Ni particles is reduced by the increase of the Ni content, so that it becomes easy for the Ni particles to contact with each other. For the aligned composites $\left(A_{m}, B_{m}\right.$ and $\left.C_{m}\right)$, the average diameters of the Ni columns are about 8, 23 and $37 \mu \mathrm{m}$ for the composite films, respectively, as shown in Figure 5. Moreover, the average distances among the Ni columns can be evaluated from over 10 measurements to be about 49,44 and $20 \mu \mathrm{m}$, respectively. Tables 2 shows further that the specific resistances of the composite films $\left(\mathrm{A}_{\mathrm{m}}, \mathrm{B}_{\mathrm{m}}\right.$ and $\mathrm{C}_{\mathrm{m}}$ ) prepared in the presence of the applied magnetic field are 4-9 orders lower in the vertical direction than in the horizontal plane. This is because the ultrafine Ni particles were aligned to form columns throughout the whole thickness of the aligned composite film when the magnetic field was applied. Thus, these columns serve as electrically conducting pathways. This anisotropy in the electrical property is a useful material characteristic that may be exploited for electronic device applications [14, 15]. However, the normal composite samples (A, B, C) prepared in the absence of a magnetic field show an isotropy in the specific resistance. This is because the ultrafine Ni particles were randomly dispersed in the epoxy matrix.

Figure 6 illustrates that the as-synthesized composite films are also very flexible. When bending the composite films, they will not be destroyed. After releasing of bending force, the composite films recover to their original shapes. In addition, repeated bending and unbending of the films up to ten times or more does not change this good behavior, thus demonstrating their excellent mechanical flexibility. Moreover, the flexibility of the samples was tested according to the standard ASTM D4338$97(2011) \mathrm{e}$. The flexibility value of the films is defined as the diameter of the smallest mandrel over which four out of five test specimens do not break. The obtained flexibility for the samples of $\mathrm{A}_{\mathrm{m}}, \mathrm{B}_{\mathrm{m}}$ and $\mathrm{C}_{\mathrm{m}}$ is respectively $4.0,5.4$ and $6.8 \mathrm{~mm}$. And the flexibility of the samples (A, B and C) is also equal to $4.0,5.4$ and $6.8 \mathrm{~mm}$, respectively. The good flexibility of these films is mainly due to the fact that the epoxy matrix modified by the flexible diamine (D-230) is flexible, as it was also done in our previous work for severe cryogenic engineering application [33]. Moreover, the specific resistance was measured for the composite films after repeated bending and unbending, and their specific resistance maintains their original values. This confirms that the composite films keep their integrity due to their excellent flexibility.

\subsection{Ferromagnetic property of Ni/epoxy composite films}

The hysteresis measurements of the composites were carried out at room temperature in an applied 


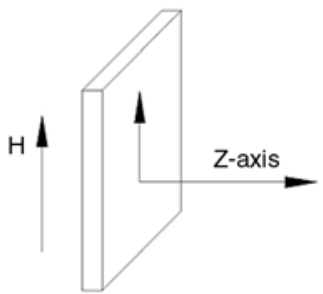

a)

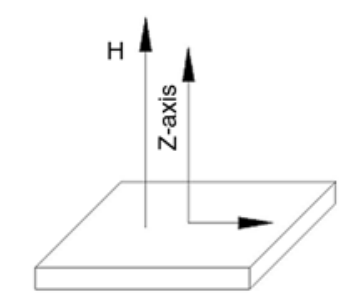

b)
Figure 7. Schematic illustration of the VSM experiment in a magnetic field with different directions: (a) horizontal and (b) vertical

magnetic field sweeping from -796 to $796 \mathrm{kA} / \mathrm{m}$ in the horizontal plane as well as in the vertical direction (Figure 7). The hysteresis loops for the aligned composite films are exhibited in Figure 8, whereby the hysteresis loops for the random composites were not presented for simplicity. The Ni/epoxy composites display a typical ferromagnetic behavior, indicating that the ferromagnetic characteristics of the ultrafine Ni particles were not altered by the preparation process of the composites. The results for the ferromagnetic properties are summarized in Table 3. It shows that the coercivity of the composite samples is about $16000-16875 \mathrm{~A} / \mathrm{m}$, which is approximately the same as for the pure ultrafine $\mathrm{Ni}$ particles. This means, the applied magnetic field has a negligible effect on the coercivity of the composite films. On the other hand, both the saturation magnetization $\left(M_{\mathrm{s}}\right)$ and remanence $\left(M_{\mathrm{r}}\right)$ of the composite films increase as the Ni content increases from 0.5 to $6.0 \mathrm{wt} \%$ (Table 3).

In order to investigate the effect of the applied magnetic field on the magnetic anisotropy of the samples, a comparison in the squareness ratio $\left(M_{\mathrm{r}} / M_{\mathrm{S}}\right)$ of the samples is presented in Table 4. For the aligned composite films, when measuring in the horizontal plane (direction), the $M_{\mathrm{r}} / M_{\mathrm{S}}$ of the samples is 0.18 , 0.23 and 0.24 , for $A_{m}, B_{m}$ and $C_{m}$, respectively; when measuring in the vertical direction, the $M_{\mathrm{r}} / M_{\mathrm{s}}$ of the three samples amounts to $0.27,0.35$ and 0.36 .
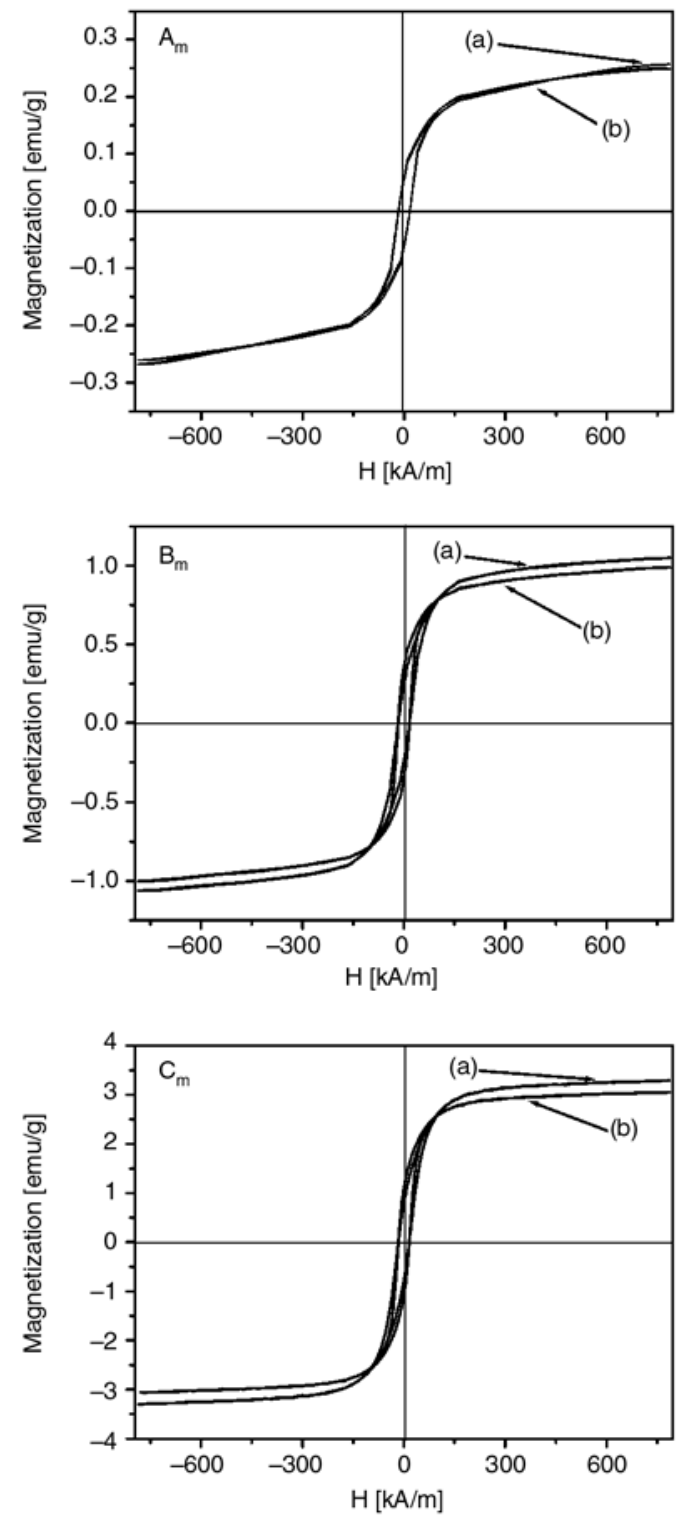

Figure 8. Hysteresis loops of the composite films $\left(\mathrm{A}_{\mathrm{m}}, \mathrm{B}_{\mathrm{m}}\right.$ and $\mathrm{C}_{\mathrm{m}}$ ): (a) horizontal and (b) vertical

The obvious difference (about 50\%) in the squareness ratio between the horizontal and vertical directions indicates that an obvious magnetic anisotropy has been induced in the aligned composite films. For the random samples (A, B and C), the $M_{\mathrm{r}} / M_{\mathrm{S}}$

Table 3. Saturation magnetization, remanence and coercivity of the Ni/epoxy composite films

\begin{tabular}{|l|c|c|c|c|c|c|}
\hline \multirow{2}{*}{ Sample code } & \multicolumn{2}{|c|}{$\begin{array}{c}\mathbf{M}_{\mathbf{s}} \\
\text { [emu/g] }\end{array}$} & \multicolumn{2}{c|}{$\begin{array}{c}\mathbf{M}_{\mathbf{r}} \\
\text { [emu/g] }\end{array}$} & \multicolumn{2}{c|}{$\begin{array}{c}\text { Coercivity } \\
{[\mathbf{A} / \mathbf{m}]}\end{array}$} \\
\cline { 2 - 7 } & Horizontal & Vertical & Horizontal & Vertical & Horizontal & Vertical \\
\hline $\mathrm{A}$ & 0.2622 & 0.2687 & 0.0603 & 0.0564 & 16687 & 16659 \\
\hline $\mathrm{B}$ & 1.1319 & 1.0433 & 0.2717 & 0.2400 & 16701 & 16670 \\
\hline $\mathrm{C}$ & 3.2646 & 3.1125 & 0.9141 & 0.8093 & 16029 & 15989 \\
\hline $\mathrm{A}_{\mathrm{m}}$ & 0.2775 & 0.2770 & 0.0500 & 0.0748 & 16571 & 16870 \\
\hline $\mathrm{B}_{\mathrm{m}}$ & 1.0550 & 0.9993 & 0.2427 & 0.3498 & 16610 & 16728 \\
\hline $\mathrm{C}_{\mathrm{m}}$ & 3.2964 & 3.0525 & 0.7911 & 1.099 & 16083 & 16115 \\
\hline
\end{tabular}


Table 4. Squareness ratio $\left(M_{\mathrm{r}} / M_{\mathrm{s}}\right)$ of the Ni/epoxy composite films

\begin{tabular}{|l|c|c|}
\hline \multirow{2}{*}{ Sample code } & \multicolumn{2}{|c|}{ Squareness ratio $\left(\mathbf{M}_{\mathbf{r}} / \mathbf{M}_{\mathbf{s}}\right)$} \\
\cline { 2 - 3 } & Horizontal & Vertical \\
\hline $\mathrm{A}$ & 0.23 & 0.21 \\
\hline $\mathrm{B}$ & 0.24 & 0.23 \\
\hline $\mathrm{C}$ & 0.28 & 0.26 \\
\hline $\mathrm{Am}$ & 0.18 & 0.27 \\
\hline $\mathrm{Bm}$ & 0.23 & 0.35 \\
\hline $\mathrm{Cm}$ & 0.24 & 0.36 \\
\hline
\end{tabular}

ratio shows approximately equal values in the two directions and thus their ferromagnetic property is isotropic.

The most promising potential application of such films is in surface mount technology [14], where comprehensive physical and mechanical properties are required for epoxy composites. To make such films, the optimal concentration of the Ni particles should be about $2.0 \mathrm{wt} \%$, and the film thickness should be below $200 \mu \mathrm{m}$ to get a high quality composite film with comprehensive physical properties.

\section{Conclusions}

In summary, optically transparent, mechanically flexible, but electrically conductive and ferromagnetic free-standing $\mathrm{Ni} /$ epoxy composite films have been prepared via a new approach. The composites are based on the incorporation of as-prepared ultrafine monodisperse Ni particles into a self-designed flexible-type transparent epoxy resin under an applied magnetic field. A water soluble poly(vinyl alcohol) film was used as the interlayer to separate epoxy resin and glass substrate so that epoxy and composite films could be easily obtained. It was shown that the Ni particles were aligned in the composite films prepared under an applied magnetic field, while the Ni particles in the reference composites prepared in the absence of an applied magnetic field showed a random filler distribution. The aligned composite films exhibited clear anisotropies in the electrical and ferromagnetic properties and a relatively higher optical transparency. Due to their comprehensive physical properties and great anisotropies in electrical and ferromagnetic properties, the aligned composite films are promising for practical applications in multifunctional electronic and electro-optical devices.

\section{Acknowledgements}

This work is financially supported by the National Natural Science Foundation of China (Nos. 10972216, 51073169 and 11002142) and Beijing Municipal Natural Science Foundation (No. 2122055). One of us, S. Y. Fu, is grateful to the Alexander von Humboldt Foundation for support of his research stay at IVW, Kaiserslautern, Germany, in March 2012.

\section{References}

[1] Liao L., Zhang Z., Yan B., Zheng Z., Bao Q. L., Wu T., Li C. M., Shen Z. X., Zhang J. X., Gong H., Li J. C., Yu T.: Multifunctional $\mathrm{CuO}$ nanowire devices: $\mathrm{p}$-type field effect transistors and CO gas sensors. Nanotechnology, 20, 085203/1-085203/8 (2009). DOI: $10.1088 / 0957-4484 / 20 / 8 / 085203$

[2] Das J., Kalinikos B. A., Barman A. R., Patton C. E.: Multifunctional dual-tunable low loss ferrite-ferroelctric heterostructures for microwave devices. Applied Physics Letters, 91, 172516/1-172516/3 (2007). DOI: $10.1063 / 1.2802577$

[3] Li S., Tay Y. Y., Sun C. Q.: Separation of lattice structural and electronic effects on physical properties with nanotechnology. Journal of Electroceramics, 21, 9198 (2008).

DOI: $10.1007 / \mathrm{s} 10832-007-9078-6$

[4] Zhang C-S., Ni Q-Q., Fu S-Y., Kurashiki K.: Electromagnetic interference shielding effect of nanocomposites with carbon nanotube and shape memory polymer. Composites Science and Technology, 67, 2973-2980 (2007).

DOI: 10.1016/j.compscitech.2007.05.011

[5] Sierros K. A., Hecht D. S., Banerjee D. A., Morris N. J., Hu L., Irvin G. C., Lee R. S., Cairns D. R.: Durable transparent carbon nanotube films for flexible device components. Thin Solid Films, 518, 6977-6983 (2010). DOI: $10.1016 /$ j.tsf.2010.07.026

[6] de Villoria R. G., Hart A. J., Wardle B. L.: Continuous high-yield production of vertically aligned carbon nanotubes on 2D and 3D substrates. ACS Nano, 5, 48504857 (2011).

DOI: $10.1021 / \mathrm{nn} 2008645$

[7] Baba K., Takase F., Miyagi M.: Magnet-optic media composed of ferromagnetic metal island films for glass and semiconductor substrates. Electronics Letters, 32, 222-224 (1996).

DOI: $10.1049 / \mathrm{el}: 19960151$

[8] Yokoyama T., Nakagawa T., Takagi Y.: Magnetic circular dichroism for surface and thin film magnetism: Measurement techniques and surface chemical applications. International Reviews in Physical Chemistry, 27, 449-505 (2008).

DOI: $10.1080 / 01442350802127608$ 
[9] Ramkumar S. M., Srihari K.: A novel anisotropic conductive adhesive for lead-free surface mount electronics packaging. Journal of Electronic Packaging, 129, 149-156 (2007).

DOI: $10.1115 / 1.2721086$

[10] Lu D., Wong C. P.: Novel conductive adhesives for surface mount applications. Journal of Applied Polymer Science, 74, 399-406 (1999).

DOI: $10.1002 /($ SICI) 1097-4628(19991010)74:2<399:: AID-APP22>3.0.CO;2-F

[11] Nam S-B., Yun G. J., Carletta J., Kim D-H., Binienda W.: A novel noncontact electromagnetic field-based sensor for the monitoring of resonant fatigue tests. Smart Materials and Structures, 20, 035005/1035005/13 (2011).

DOI: $10.1088 / 0964-1726 / 20 / 3 / 035005$

[12] Adachi N., Yogo K., Ota T., Takahashi M., Ishiyama K.: Magneto-optical effect and ferromagnetic resonance of $\mathrm{Bi}-\mathrm{Fe}$ garnet for high frequency electromagnetic sensor. Journal of Applied Physics, 109, 07A506/107A506/3 (2011).

DOI: $10.1063 / 1.3556709$

[13] Zhang B., Xie C., Hu J., Wang H., Gui Y.: Novel 1-3 metal nanoparticle/polymer composites induced by hybrid external fields. Composites Science and Technology, 66, 1558-1563 (2006).

DOI: $10.1016 /$ j.compscitech.2005.11.020

[14] Jin S., Tiefel T. H., Wolfe R., Sherwood R. C., Mottine J. J. Jr.: Optically transparent, electrically conductive composite medium. Science, 255, 446-448 (1992). DOI: $10.1126 /$ science. 255.5043 .446

[15] Sancaktar E., Dilsiz N.: Anisotropic alignment of nickel particles in a magnetic field for electronically conductive adhesives applications. Journal of Adhesion Science and Technology, 11, 155-166 (1997). DOI: $10.1163 / 156856197 X 00273$

[16] Kim M. J., Yoo J-B.: Electrical properties of transparent conductive CNT composite films. Electronic Materials Letters, 4, 57-61 (2008).

[17] Harada M., Ueda A., Miyazaki H., Ochi M.: Mechanical properties of the flexible-type epoxy/clay nanocomposites prepared by slurry method. Journal of Applied Polymer Science, 113, 2256-2263 (2009).

DOI: 10.1002 /app.30396

[18] Taucher-Mautner W., Kordesch K.: Studies of pasted nickel electrodes to improve cylindrical nickel-zinc cells. Journal of Power Sources, 132, 275-281 (2004). DOI: 10.1016/j.jpowsour.2004.01.032

[19] Zeng W., Tan S. T.: Preparation and EMI shielding properties of nickel-coated PET fiber filled epoxy composites. Polymer Composites, 27, 24-29 (2006). DOI: $10.1002 /$ pc.20094

[20] Park J-M., Wang Z-J., Kwon D-J., Jang J-H., DeVries K. L.: Interfacial evaluation and hydrophobicity of multi-functional Ni-nanopowder/epoxy composites for self-sensing and actuation. Smart Materials and Structures, 19, 124006/1-124006/8 (2010). DOI: $\underline{10.1088 / 0964-1726 / 19 / 12 / 124006}$
[21] Shinko Denki Kogyo K. K.: Lead frame for resinmoulded semiconductor device - has connection between holes etched into front and rear surfaces of element mound die pad. Japanese Patent, JP3006851-A (1991).

[22] Li Y. Q., Fu S. Y., Yang Y., Mai Y. W.: Facile synthesis of highly transparent polymer nanocomposites by introduction of core-shell structured nanoparticles. Chemistry of Materials, 20, 2637-2643 (2008).

DOI: $\underline{10.1021 / \mathrm{cm} 7033307}$

[23] Barletta M., Bellisario D.: Manufacture and characterization of free-standing epoxy-polyester films. Progress in Organic Coatings, 70, 259-272 (2011).

DOI: 10.1016/j.porgcoat.2010.09.029

[24] Rath S. K., Chavan J. G., Sasane S., Jagannath, Patri M., Samui A. B., Chakraborty B. C.: Two component silicone modified epoxy foul release coatings: Effect of modulus, surface energy and surface restructuring on pseudobarnacle and macrofouling behavior. Applied Surface Science, 256, 2440-2446 (2010).

DOI: $10.1016 /$ j.apsusc.2009.10.084

[25] Afzal A., Siddiqi H. M.: A comprehensive study of the bicontinuous epoxy-silica hybrid polymers: I. Synthesis, characterization and glass transition. Polymer, 52, 1345-1355 (2011).

DOI: 10.1016/j.polymer.2011.01.046

[26] Posner R., Santa M., Grundmeier G.: Wet- and corrosive de-adhesion processes of water-borne epoxy film coated steel. Journal of the Electrochemical Society, 158, C29-C35 (2011).

DOI: $10.1149 / 1.3525239$

[27] Santa M., Posner R., Grundmeier G.: Wet- and corrosive de-adhesion processes of water-borne epoxy film coated steel. Journal of the Electrochemical Society, 158, C36-C41 (2011).

DOI: $10.1149 / 1.3525240$

[28] Barletta M., Gisario A.: The role of the substrate in micro-scale scratching of epoxy-polyester films. Applied Surface Science, 257, 4449-4463 (2011). DOI: $10.1016 /$ j.apsusc.2010.12.090

[29] Maiti P. K.: Polymer-insulating material for rated electrical applications. Journal of Applied Polymer Science, 117, 2310-2315 (2010).

DOI: 10.1002/app.32071

[30] Kostrzewa M., Hausnerova B., Bakar M., Siwek E.: Effects of various polyurethanes on the mechanical and structural properties of an epoxy resin. Journal of Applied Polymer Science, 119, 2925-2932 (2011). DOI: 10.1002/app.32974

[31] Ratna D., Chakraborty B. C., Dutta H., Banthia A. K.: Nanoreinforcement of flexible epoxy using layered silicate. Polymer Engineering and Science, 46, 16671673 (2006). DOI: $10.1002 /$ pen.20628 
[32] Nazir T., Afzal A., Siddiqi H. M., Ahmad Z., Dumon M.: Thermally and mechanically superior hybrid epoxysilica polymer films via sol-gel method. Progress in Organic Coatings, 69, 100-106 (2010). DOI: 10.1016/j.porgcoat.2010.05.012

[33] Yang G., Fu S-Y., Yang J-P.: Preparation and mechanical properties of modified epoxy resins with flexible diamines. Polymer, 48, 302-310 (2010).

DOI: $10.1016 /$ j.polymer.2006.11.031

[34] Duan C-G., Velev J. P., Sabirianov R. F., Mei W. N., Jaswal S. S., Tsymbal E. Y.: Tailoring magnetic anisotropy at the ferromagnetic/ferroelectric interface. Applied Physics Letters, 92, 122905/1-122905/3 (2008).

DOI: $\underline{10.1063 / 1.2901879}$
[35] Wei H. X., Qin Q. H., Wen Z. C., Han X. F., Zhang XG.: Magnetic tunnel junction sensor with $\mathrm{Co} / \mathrm{Pt}$ perpendicular anisotropy ferromagnetic layer. Applied Physics Letters, 94, 172902/1-172902/3 (2009).

DOI: $10.1063 / 1.3126064$

[36] Shen X-J., Yang J-P., Liu Y., Luo Y-S., Fu S-Y.: Facile surfactant-free synthesis of monodisperse Ni particles via a simple solvothermal method and their superior catalytic effect on thermal decomposition of ammonium perchlorate. New Journal of Chemistry, 35, 1403-1409 (2011).

DOI: $10.1039 / \mathrm{c} 0 \mathrm{nj} 00987 \mathrm{c}$

[37] Liu Q., Liu H., Han M., Zhu J., Liang Y., Xu Z., Song Y.: Nanometer-sized nickel hollow spheres. Advanced Materials, 17, 1995-1999 (2005).

DOI: $\underline{10.1002 / \mathrm{adma} .200500174}$ 